\title{
Contagio entre las bolsas de estados unidos y las de América Latina: el caso de la crisis financiera de 2008
}

\section{Contagion between the united states and latinamerican stock markets: The case of the financial crisis of 2008}

\section{Domingo Rodríguez Benavides*}

Universidad Autónoma Metropolitana, México

Recibido el 17 de agosto de 2017; aceptado el 9 de abril de 2018

Disponible en Internet el:10 de junio de 2019

\section{Resumen}

En este trabajo empleamos la prueba de contagio financiero sugerida por Hatemi y Hacker (2005) con el fin de probar la hipotesis de contagio en los principales mercados accionarios de America Latina por parte de los mercados accionarios de Estados Unidos en la crisis del subprime. Dicha prueba se basa en el método de remuestreo con bootstrap, el cual se considera robusto a la ausencia de normalidad y de una creciente volatilidad (heterocedasticidad), caracteristicas inherentes a los mercados financieros particularmente en periodos de crisis. Los resultados muestran evidencia de contagio financiero en la crisis subprime de 2008 de los principales indices accionarios de Estados Unidos a algunos de los principales mercados financieros de Latinoamerica.

Código JEL: F36, G15, C22

Palabras clave: Contagio financiero; Beta; Método de Bootstrap

\footnotetext{
*Autor para correspondencia. Correo electrónico dorobe@azc.uam.mx(D. Rodríguez Benavides).

La revisión por pares es responsabilidad de la Universidad Nacional Autónoma de México. 


\begin{abstract}
In this paper we use the financial contagion test suggested by Hatemi and Hacker (2005) in order to test the hypothesis of contagion in the main equity markets of Latin America by the US equity markets in the subprime crisis. This test is based on the bootstrapping method, which is considered robust to the absence of normality and increasing volatility (heteroskedasticity), characteristics inherent to financial markets, particularly during periods of crisis. The results show evidence of financial contagion in the subprime crisis of 2008 from the main US stock indices to some of the main financial markets in Latin America.
\end{abstract}

JEL codes: F36, G15, C22

Keywords: Financial contagion; Beta; Bootstrap method

\title{
Introducción
}

Durante las últimas décadas, algunos países de América Latina como Chile, Bolivia y México, como muchos otros países emergentes y en desarrollo, han hecho notables esfuerzos por liberalizar sus fronteras al comercio y flexibilizar el intercambio de sus divisas. A la par de estos procesos, algunos de ellos han avanzado hacia una mayor integración en el sistema financiero internacional. En varios de estos países se ha promovido la entrada de capitales al mismo tiempo que se han relajado los controles para su salida, Agosin y French-Davis (1993). La razón de estas políticas se basa en la expectativa general de que la liberalización comercial y financiera impulsaría la actividad económica reforzando la competitividad, abriendo nuevos mercados de exportación, atrayendo la inversión extranjera directa y estimulando el ahorro y la inversión nacional. Las reformas que han impulsado estos procesos han propiciado una mayor cooperación con los países desarrollados, en particular con los Estados Unidos y la Unión Europea, desembocando en algunos casos en la conformación de acuerdos de libre comercio o de acuerdos de asociación.

Sin embargo, no hay una visión unanime acerca de las consecuencias de esta integración financiera, Khallouli y Sandretto (2012). Por el contrario, otros subrayan el hecho de que una mayor integración en los mercados financieros internacionales aumenta la vulnerabilidad a las influencias provenientes del exterior, en particular a las inversiones en los movimientos de capitales internacionales, lo que se denomina riesgo de "contagio", y propicia que estos países pueden verse cada vez mas afectados por los choques externos negativos, Colins y Biekpe (2003) y Bekaert et al. (2005).

La crisis del subprime, por ejemplo, no se limitó realmente a los mercados hipotecarios de los Estados Unidos. Como resultado de la titularización, la crisis se extendió a todo el mercado financiero, no sólo en Estados Unidos, sino también a todos los países desarrollados 
(Horta et al. 2008). La magnitud y profundidad de esta crisis mundial, considerada por algunos como la más severa desde la crisis de 1929, así como la posibilidad para algunos países de ser contagiados y experimentar las consecuencias de sus efectos han hecho resurgir el interés en el contagio financiero y en sus mecanismos de propagación.

Hay una amplia literatura sobre contagio financiero y sobre sus canales pero no existe una definición universalmente aceptada de contagio en la literatura, sin embargo historicamente se le ha asociado con un incremento en los vinculos entre mercados después de un choque a un país o un grupo de países, Forbes y Rigobon (2002) y Hatemi-J y Hacker (2005).

Existen diversas formas de definir contagio financiero. Por ejemplo, Forbes y Rigobon (2002) lo define como un incremento significativo de los vinculos entre los mercados después de un choque a un país o grupo de países. De acuerdo con esta definición, no ocurre contagio si dos mercados muestran un alto grado de interdependencia financiero durante los periodos de estabilidad y de crisis, Fuchun (2009). Otras formas de contagio son como el aumento de la probabilidad de crisis, que va más allá de los vínculos en los fundamentos, y el rápido aumento de los co-movimientos entre los mercados durante los episodio de crisis, Forbes y Rigobon (2012), Bekaert, Harvey y Lumsdaine(2002).

Empiricamente, una forma de probar contagio es a través de los coeficientes de correlación cruzados entre distintos mercados geograficos, King y Wadhwani (1990), Lee y Kim (1993), Calvo y Reinhart (1996), Forbes y Rigobon (2002), Hon et al. (2004), Pretorius y Beer (2004). Hatemi-J y Hacker (2005) mencionan otros métodos para probar contagio, los cuales son: $(i)$ el uso de matrices de varianza-covarianza en los modelos ARCH y GARCH con el objetivo de investigar potenciales mecanismos de transmisión; (ii) los que analizan cambios en vectores de cointegración entre países; y (iii) los que investigan si una variedad de factores afectan la susceptibilidad del mercado a las crisis financieras.

Se ha demostrado que la estimación de los coeficientes de correlación poblacionales entre variables financieras de distintos mercados geograficos está sesgada si la volatilidad es mayor para algún periodo de tiempo de los datos sujetos a comparación, Forbes y Rigobon (2012). En este trabajo empleamos un método alternativo para probar contagio financiero, propuesto por Hatemi-J y Hacker (2005), quienes en lugar de probar contagio entre mercados financieros a través de la comparación de coeficientes de correlación, entre periodos con distinta volatilidad, sugieren averiguar si hay o no cambio en el coeficiente de la pendiente en una regresión de una variable financiera de un mercado contra una variable financiera de otro mercado en el periodo en el que inicia un periodo de mayor volatilidad. Hatemi-J y Hacker (2005) investigaron las propiedades de la prueba a través de simulaciones Monte Carlo en virtud de que dificilmente se cumplen los supuestos clásicos que subyacen al modelo de regresión con este tipo de variables. Otra innovación a la literatura empirica sobre el tema de nuestro trabajo es que analizamos la posibilidad de contagio financiero a los mercados accionarios 
de America Latina a partir de distintos indices accionarios de Estados Unidos considerados como los mas representativos.

\section{Breve Revisión de la Literatura Empírica}

Bodart y Candelon (2009) proponen una medida de contagio a través de la prueba de causalidad en el dominio de frecuencia desarrollada por Breitung y Candelon (2006). Bodart y Candelon (2009) definen el contagio financiero como un incremento temporal y significativo en los vinculos entre los mercados después de un shock. Una de las principales ventajas de la prueba propuesta por Bodart y Candelon (2009) es que este permite diferenciar claramente entre cambios temporales y permanentes en los vinculos entre mercados, de los cuales a los primeros son considerados como evidencia de contagio, mientras que los segundos son simplemente una medida de interdependencia entre los mercados. Con este método prueban la existencia de contagio entre varios mercados de Latinoamerica y de Asia durante las crisis financieras internacionales de 1994 y 1997. Sus resultados muestran evidencia de contagio en las dos crisis bajo estudio, adicionalmente su enfoque muestra que durante la crisis Asiatica, tanto el contagio como la interdependencia fueron responsables de que se fortalecieran los vinculos entre los mercados analizados, lo cual no se observa en la crisis del Tequila. De igual forma destacan que efectos de derrama de esas crisis han estado confinados geograficamente a la región donde ocurrió el choque (shock). También encuentran soporte de que el contagio es mas regional que global como lo sugieren los trabajos de Glick y Rose (1999), Kaminsky y Reinhart (2000).

Neaime (2002) estudió una mezcla de países del Medio Oriente, del Norte de Africa y del Consejo de Cooperación del Golfo (CCG) durante el período 1990-2000. Sus resultados muestran una creciente integración financiera regional en los países del CCG y una fuerte sensibilidad a los choques unidireccionales provientes de Estados Unidos y el Reino Unido en los países de la región del Oriente Medio y del Norte de Africa. Erdal y Gunduz (2001) investigaron la interdependencia de la Bolsa de Estambul con los mercados de renta variable del G-7 y los mercados de valores de Israel, Jordania, Egipto y Marruecos, antes y después de la crisis asiática. Erdal y Gunduz (2002) rechazan la hipótesis de vínculos significativos entre los mercados del Medio Oriente y del Norte de Africa basados en las pruebas de causalidad de Granger y encontraron evidencia de un sólo vector de cointegración entre la Bolsa de Estambul y los mercados del G-7.

Khallouli y Sandretto (2012) investigan si la crisis subprime registrada en Estados Unidos contaminó los países del Medio Oriente y del Norte de Africa. Khallouli y Sandretto (2012) intentan encontrar evidencia de "contagio puro" en lugar de "shift-contagion", para tal fin 
ellos estiman un modelo EGARCH Markov-Switching, propuesto por Henry (2009), para determinar si los mercados accionarios bajo estudio se contaminaron. Sus resultados proveen evidencia de una persistente recesión caracterizada por régimenes de alta y baja varianza, los cuales coinciden con lo que ellos denominan la tercera fase de la crisis subprime. Adicionalmente, encuentran evidencia de contagio en la media y en la varianza de los mercados accionarios del Medio Oriente y del Norte de Africa ocasionado por el mercado accionario de los Estados Unidos.

Dimitriou, Kenourgios y Simos (2013) investigan los efectos de contagio de la crisis financiera global a través de un modelo multivariado de Potencias Asimetricas Fraccionalmente Integrado (FIAPARCH) de correlación dinámicas condicionales (DCC) en los cinco mas importantes mercados emergentes denominados Brasil, Rusia, India, China y Africa del Sur (BRICS) y los Estados Unidos en el periodo 1997-2012. Dimitriou et al. (2013) identifican tanto la duración como las fases de la crisis con enfoques económicos y estadísticos. La evidencia empirica que brindan los autores no confirma efecto contagio para la mayoría de los BRICS durante etapas tempranas de la crisis, sugiriendo signos de aislamiento y desacoplamiento. No obstante, encuentran que los vinculos resurgen después del colapso de Lehman Brothers, lo cual es atribuido por ellos a un cambio en el apetito del riesgo de los inversores. Adicionalmente, muestran evidencia de que las correlaciones entre los BRICS y Estados Unidos se ha incrementado desde principios del 2009 lo cual pone de manifiesto que la dependencia es mayor en los periodos alcistas que en los bajistas.

Fedderke y Marinkov (2016) proponen una prueba de diagnostico para determinar si los choques financieros se deben a la propagación de choques idiosincraticos originados en un solo país (o grupo de países), o son más bien un reflejo de la interdependencia del mercado debido a factores comunes entre mercados. La prueba propuesta por Fedderke y Marinkov (2016) está dada por la razón, I , obtenida por la razón de los coeficientes de correlación condicional e incondicional entre los mercados. Ellos demuestran analiticamente que su estadístico de prueba es robusto a una serie de factores como son la heterocedasticidad debido a la volatilidad condicional del mercado, al impacto de variables omitidas y al impacto de la endogeneidad entre los mercados. Los resultados de las pruebas aplicados a la crisis asiatica y del subprime con la prueba I sugieren que la propagación de choques fue predominantemente debido a fundamentales comunes, mientras que en la crisis Europea la propagación de choques es idiosincratica centrados en Chipre, Crecia y Latvia.

Dentro de los trabajos que se han realizado sobre el tema para los países de America Latina se encuentra el de Chirinos (2013), quien utiliza las cópulas y los eventos extremos para medir la interdependencia de los mercados de America Latina. A partir de la teoría de los valores extremos encuentra que los choques negativos se trasmiten con mayor fuerza que los positivos en los países de la región. 
Bejarano, Gomez, Melo y Torres (2015) emplean el modelo modelo GARCH Multivariado de Correlaciones Dinámicas Condicionales para probar contagio en los mercados financieros de America Latina de los choques provenientes de los Estados Unidos y Europa en el periodo comprendido entre el 4 de julio de 2001 y el 30 de diciembre de 2013, sus resultados muestran evidencia de dos periodos de contagio, el primero corresponde a la crisis subprime de Estados Unidos y el segundo al periodo de turbulencia de los bonos soberanos de Europa registrado entre 2011 y 2012.

Romero, Bonilla, Benedetti y Serletis (2015) emplean la prueba de bicorrelación portmanteau de Hinich (1996) para representar eventos nolineales detectados en los mercados accionarios de America Latina. Ellos identifican el comienzo, el final, la intensidad y la persistencia de episodios no lineales. Los seis episodios que identifican en el período de enero de 1994 a noviembre de 2012 se encontraron contemporáneos de las crisis financieras internacionales, lo que les permite especular que el contagio causado por las crisis financieras induce dependencias no lineales. Del mismo modo observan una estructura no lineal sistemática en la serie de rendimiento del índice bursátil que se ha asociado con la falta temporal de eficiencia del mercado.

Mollah, Quoreshi y Zafirov (2016) encuentran evidencia de contagio en mercados desarrollados y emergentes durante las crisis mundiales y de la Eurozona a través de los índices diarios MSCI denominados en dólares estadounidenses de cincuenta y cinco mercados de renta variable para el período 2003-2013. Sus resultados muestran que el contagio se extendió desde los Estados Unidos a los mercados mundiales durante ambas crisis. De igual forma identifican que la transferencia de riesgo bancario entre los Estados Unidos y otros países es el canal de transmisión clave para las correlaciones entre países.

Polanco, Fernández y Neumann y Faria(2018)efectuan un análisis de los índices bursátiles periféricos de la Unión Europea (denominados PIIGS) con el índice S\&P Europe 350 (SPEURO), como mercado europeo de referencia, durante los periodos de pre-crisis (2004-2007) y de crisis (2008-2011). Ellos calculan una correlación espectral con ventanas moviles para los rendimientos accionarios y aplican pruebas no lineales de causalidad de Granger a los coeficientes de la descomposición espectral de los rendimientos. Sus resultados muestran que la correlación es más fuertepara el periodo de crisis que para el periodo previo a la crisis y que los índices bursátiles de Portugal, Italia y España estuvieron más interconectados entre sí durante la crisis que con SPEURO. De igual forma encontraron que el mercado bursátil de Portugal es el miembro de los PIIGS que es más sensible y vulnerable, mientras que el mercado de valores de Grecia tiende a alejarse del mercado europeo de referencia desde la crisis financiera de 2008 hasta 2011. Las pruebas de causalidad no lineal indican que en las primeras tres escalas de wavelets (intrasemanal, semanal y quincenal) el número de causalidades unidireccionales y bidireccionales es mayor durante la crisis que en el período previo a 
la crisis, lo cual es atribuido por ellos al contagio financiero. Además, el análisis de causalidad muestra que la dirección de la causa-efecto de Granger para los períodos previos a la crisis y crisis no es invariable a las escalas de tiempo consideradas, y que las direcciones de causalidad entre los mercados de valores estudiados no parecen tener un efecto de dirección preferencial.

\section{Metodología Econométrica}

De acuerdo con Hatemi-J y Hacker (2005) con el fin de probar contagio de una crisis en el mercado que genera $X$ al mercado generado por $Y$, consideran dos periodos: un periodo sin crisis y un periodo con crisis, siendo $X$ y $Y$ variables financieras en dos mercados geograficos diferentes. No obstante, las variable son generadas en forma dependiente cuya volatilidad se incrementa en los periodos de crisis en comparación de los periodos sin crisis. Para tal efecto,Hatemi-J y Hacker (2005) parten de la idea de un proceso generador de datos en el que la variable $Y$ se determina tanto por el nivel de $X$ como por su variabilidad además de un término de error independiente.

Es decir, la variabilidad de $Y$ se basa sobre la variabilidad de $X$ como de un término independiente de error. Para estos autores hay evidencia de contagio si el efecto de los cambios en $X$ sobre los cambios en $Y$ se incrementa signficativamente durante el periodo de crisis. En otras palabras hay evidencia de contagio si el coeficiente de la pendiente entre las variables, en una regresión de $Y$ sobre $X$, se incrementa significativamente en el periodo de alta volatilidad. De manera mas explicta, Hatemi-J y Hacker (2005) asumen el siguiente proceso generador de datos que esta dado por las siguientes ecuaciones:

$$
\begin{aligned}
& X_{L, t}=c_{L}+\varepsilon_{X L, t}, \quad \varepsilon_{X L, t}: f\left(0, \sigma_{X L, t}^{2}\right) \\
& Y_{L, t}=\alpha_{L}+\beta_{L} X_{L, t}+\varepsilon_{Y L, t}, \quad \varepsilon_{Y L, t}: \quad f\left(0, \sigma_{Y L, t}^{2}\right)
\end{aligned}
$$

$$
\begin{aligned}
& X_{H, t}=c_{H}+\varepsilon_{X H, t}, \quad \varepsilon_{X H, t}: f\left(0, \sigma_{X H, t}^{2}\right) \\
& Y_{H, t}=\alpha_{H}+\beta_{H} X_{H, t}+\varepsilon_{Y H, t}, \quad \varepsilon_{Y H, t}: f\left(0, \sigma_{Y H, t}^{2}\right)
\end{aligned}
$$


$Y$ significa la variable dependiente, $X$ es la variable independiente (con media igual a $c$ ), y $\varepsilon$ es el término de error. $\alpha_{\text {y }} \boldsymbol{\beta}$ son los coeficientes de los parametros en la ecuación de regresión. Los subindices $L_{\mathrm{y}} H$ representan los periodos de baja y alta volatilidad, respectivamente, de los cuales este ultimo periodo es ocasionado por la presencia de una crisis. Los terminos de las varianzas $\sigma_{X L, t}^{2}, \sigma_{Y L, t}^{2}, \sigma_{X H, t}^{2}$ y $\sigma_{Y H,}^{2}$ tienen como subindice el tiempo permitiendo así la posibilidad de heterocedasticidad en cada una de las variables aleatorias asociadas.

La prueba de contagio propuesta por Hatemi-J y Hacker (2005) consiste en probar la siguiente hipotesis nula:

$$
H_{0}: \beta_{2}=0
$$

En el siguiente modelo de regresión:

$$
Y_{t}=\alpha_{1}+\alpha_{2} D_{t}+\beta_{1} X_{t}+\beta_{2} D_{t} X_{t}+e_{t}
$$

Donde $D_{1}$ es una variable dummy que es igual a cero para el periodo de baja volatilidad (L) y toma el valor de 1 en el periodo de alta volatilidad (H). La regresión planteada en (4) se estima para la muestra completa de valores, es decir para ambos periodos de volatilidad. El coeficiente $\beta_{2}$ representa el cambio en la pendiente de la regresión, por lo que la hipótesis nula de que $\beta_{2}$ es igual a cero implica que no hay cambio en la relación entre $X$ e $Y$, lo que a su vez no significa ningún contagio de $X$ a $Y$. Esta hipótesis nula se prueba a través del estadístico ${ }^{t}$ asociado con $\beta_{2}$, Hatemi-J y Hacker (2005). El procedimiento contempla la posibilidad de que se infrinja el supuesto de homocedasticidad en virtud de que la varianza del error puede diferir entre los periodos de baja y alta volatilidad d $\left(\sigma_{Y, t}^{2} \neq \sigma_{Y H, t}^{2}\right)$.

Con base en Hatemi-J, Roca y Al-Shayeb (2014) y con el fin de ilustrar el procedimiento de bootstrap empleado, planteamos el modelo expuesto en (4) en términos de notación matricial de la siguiente forma:

$$
Y=\beta X+e
$$

donde:

$$
Y=\left[\begin{array}{c}
Y_{1} \\
Y_{2} \\
\mathrm{M} \\
Y_{t}
\end{array}\right] \text { es un vector }(T \times 1)
$$




$$
\begin{aligned}
X & =\left[\begin{array}{cccc}
1 & D_{1} & X_{1} & D_{1} X_{1} \\
1 & D_{2} & X_{2} & D_{2} X_{1} \\
\mathrm{M} & \mathrm{M} & \mathrm{M} & \mathrm{M} \\
1 & D_{T} & X_{t} & D_{3} X_{1}
\end{array}\right] \text { es una matriz de }(T \times 4) . \\
\beta & =\left[\begin{array}{llll}
\alpha_{1} & \alpha_{2} & \beta_{1} & \beta_{2}
\end{array}\right] \text { es un vector de }(4 \times 1) ; \mathrm{y} \\
e & =\left[\begin{array}{c}
e_{1} \\
e_{2} \\
\mathrm{M} \\
e_{t}
\end{array}\right] \text { es un vector }(T \times 1)
\end{aligned}
$$

Como es conocido, el vector de parametros se puede estimar a través de MCO de la siguiente forma:

$$
\beta=\left(X^{\prime} X\right)^{-1} X^{\prime} Y
$$

La implementación del método de bootstrap consiste en las siguientes 4 etapas:

1. Generar las series de tiempo $Y^{*}$ y $X^{*}$ a través del método de bootstrap con reemplazo, esto es generar

$$
Y^{*}=\left\{Y_{1}^{*}, Y_{2}^{*}, \cdots, Y_{t}^{*}\right\}, \quad Y_{j}^{*} \in Y \forall j
$$

donde $j=1, \mathrm{~L}, T$. Con $T$ es el tamaño de la muestra del bootstrap. De manera analoga:

$$
X^{*}=\left\{X_{1}^{*}, X_{2}^{*}, \cdots, X_{t}^{*}\right\}, \quad X_{j}^{*} \in X \forall j
$$

2. El vector de parametros $(\boldsymbol{\beta})$ se estima con las series resultantes del remuestreo vía bootstrap de la siguiente manera:

$$
\beta=\left(X^{* 1} X^{*}\right)^{-1} X^{* 1} Y^{*}
$$

3. Las fases 1 y 2 se iteran $N$ veces, Hatemi-J et al (2014) sugieren que $N=10,000$ y es el numero de iteracciones que empleamos en las estimaciones. 
4. Por último, a partir de los coeficientes estimados por el bootstrap $\left(\beta^{* *}\right)$ se obtiene su mediana. Hatemi-Jet al (2014) emplean la mediana en lugar de la media argumentando que la primera es robusta a la presencia de outliers.

Este enfoque de bootstrap se utiliza también para implementar las pruebas de significancia estadística de cada parametro.El valor-p de cada parametro de prueba que está en la forma $H_{0}: r \mathrm{~b}=0$ se obtiene de igual forma por el método de bootstrap. Aquí $r$ es un vector (1x4) que clasifica las restricciones impuestas por la hipotesis nula.

El valor-p del bootstrap para está hipotesis se calcula del siguiente modo. Si la mediana para $r B^{*}$ es un número positivo, entonces el valor-p es el porcentaje de elementos de la distribución resultante del bootstrap para $r B^{*}$ que son negativos más aquellos que son mayores que dos veces la mediana. Sin embargo, si la mediana para $r B^{*}$ es menor que cero, entonces el valor-p es el porcentaje de la distribución resultante del bootstrap para $r B^{*}$ que son positivos más el porcentaje de elementos en $r B^{*}$ que son menores que el doble de la mediana, Hatemi-J, Roca y Al-Shayeb (2014).El punto de corte de dos veces la mediana de $r B^{*}$ genera valores-p, que son comparables a las pruebas simétricas de dos lados en un enfoque de prueba de hipótesis tradicional, como lo indican Hatemi-J y Hacker (2005).

\section{Datos y Resultados Empiricos}

Con la finalidad de evaluar la hipotesis de contagio financiero de los mercados accionariosmás representativos deEstados Unidos hacia los principales mercados de America Latina en el caso de la crisis subprime, se tomaron datos diarios del precio de cierre del 12 de octubre de 2007 al 16 de junio de 2009, para un total de 438 observaciones, en moneda local y en dólares, de los siguientes mercados accionarios: Chile (IPSA), Colombia (IGBC), Argentina (MERVAL), Brasil (IBOV), México (IPyC) y de losEstados Unidos (NASDAQ, DJIA y S\&P 500). Se obtuvieron los rendimientos diarios $\left(r_{i i}\right)$ de cada mercado $(i)$ a través de la primera diferencia logaritmica del precio de cierre de los indices anteriores, es decir a través de $r_{i t}=\ln \left(p_{i t} / p_{i t-1}\right)$. La evolución de los rendimientos de los indices accionarios en el periodo de estudio se presentan en las figuras 1, en moneda local, y en dólares en la figura 2. A grandes rasgos se muestra un periodo de relativa estabilidad a finales de 2007 y hasta mediados de septiembre de 2008, con excepción de los primeros meses de 2008 para algunos mercados donde se presenta un periodo corto de volatilidad financiera, desde luego esta es mucho mayor y mas notoria a partir de mediados de septiembre de 2008 en adelante. A pesar de que no hay consenso en la fecha en que inicio la crisis de los vencimientos subprime, por ejemplo Lee (2012) ubica el inicio de la crisis en julio de 2007, algunos otros ubican su inicio con la notable caida del Dow 
Jones de finales de septiembre de 2007 hasta mediados de septiembre de 2008 con la quiebra de Lehman Brothers, aquí tomamos como fecha de inicio de la crisissubprime a mediados de septiembre de 2008 con el anuncio de esta importante institución financiera. De esta manera, la variable dummy $D_{t}$ toma el valor de 1 a partir del 15 de septiembre de 2008 hasta el final de la muestra del periodo analizado y el valor de cero para los otros valores previos a esta fecha.

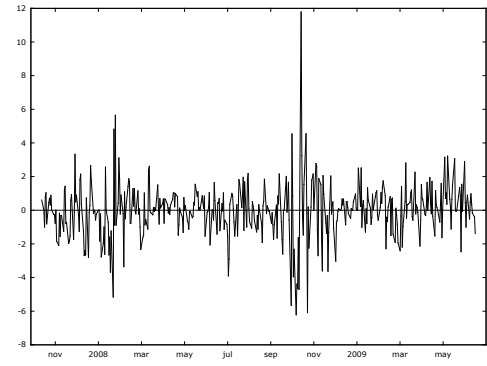

IPSA

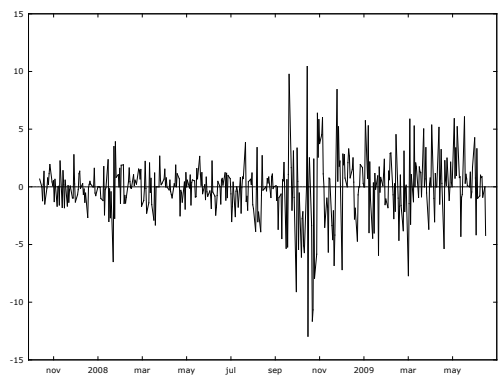

MERVAL

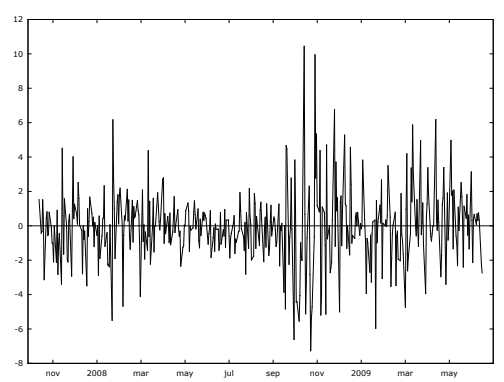

IPyC

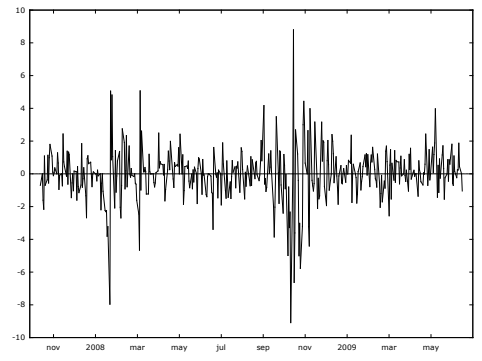

IGBC

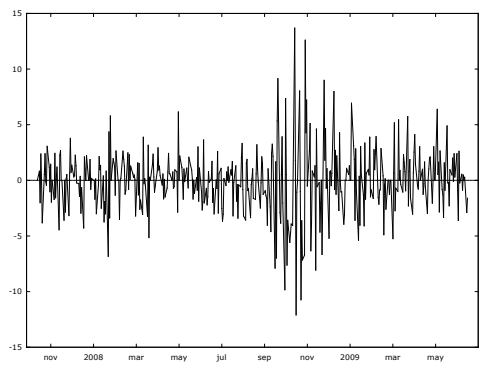

IBOV

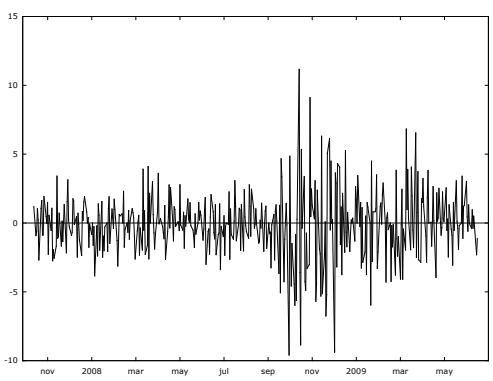

NASDAQ 


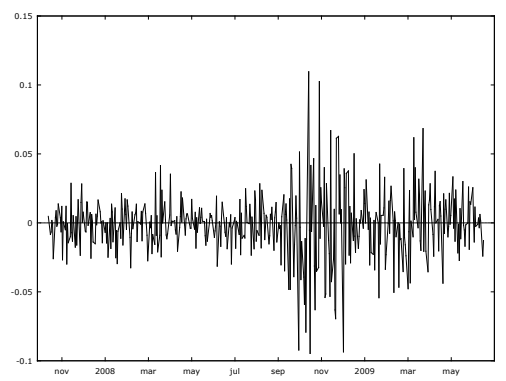

DJIA

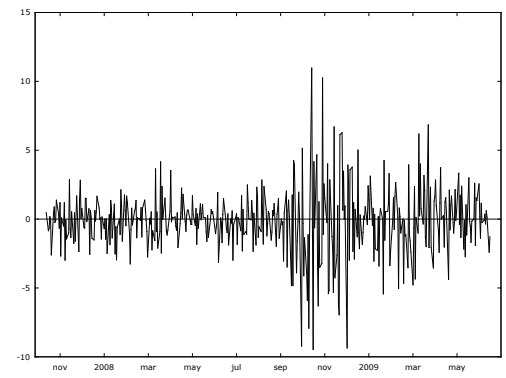

S\&P 500

Figura 1. Rendimientos diarios de los mercados accionarios de América Latina del:

12 de octubre de 2007 al 16 de junio de 2009 (Datos en moneda local).

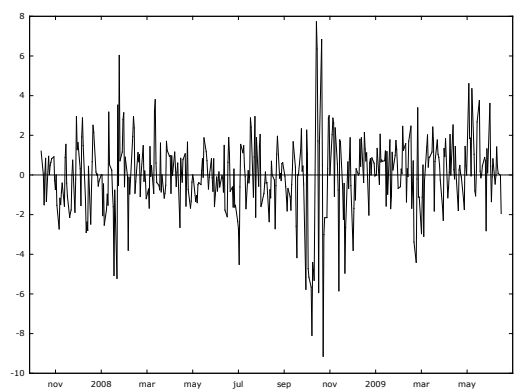

IPSA

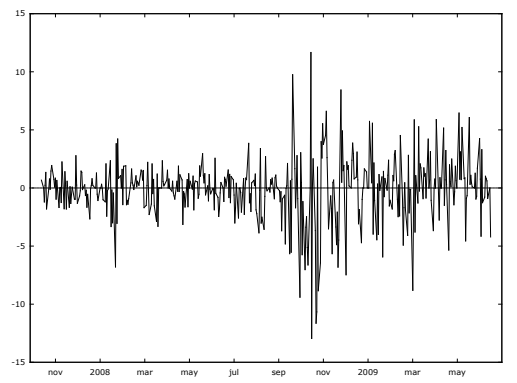

MERVAL

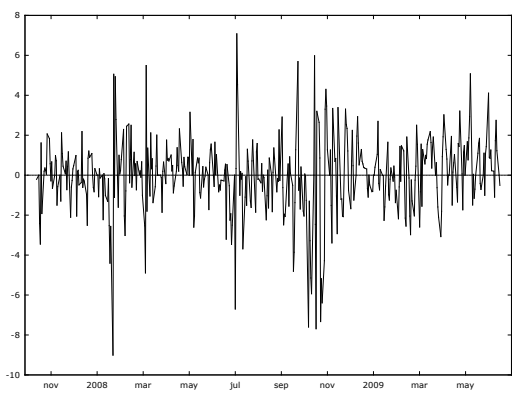

IGBC

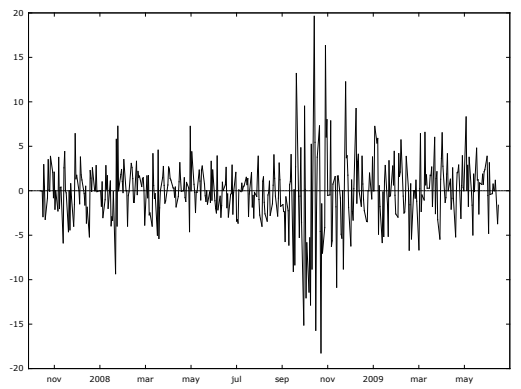

IBOV 


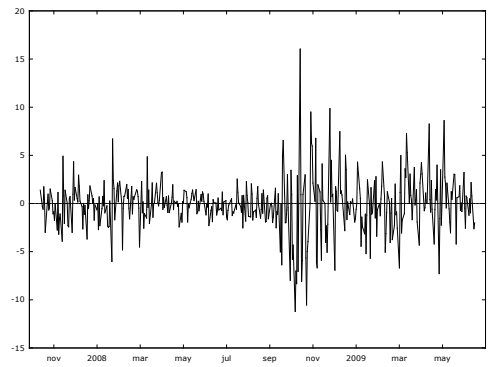

IPyC

Figura 2. Rendimientos diarios de los mercados accionarios de América Latina del: 12 de octubre de 2007 al 16 de junio de 2009 (Datos en dólares).

Los resultados del modelo planteado en la ecuación (4) para efectuar la prueba de contagio de la crisis subprime se presentan en las tablas 1,2 y 3 tomando al NASDAQ, al DJIA y al S\&P 500 como el mercado accionario independiente, respectivamente, o bien del cual el resto de los mercados accionarios dependen, para los datos en moneda local y en las tablas 4, 5 y 6 para los rendimientos denominados en dólares, respectivamente. Cuando se toma como variable dependiente a los rendimientos accionarios del NASDAQ, los resultados de la prueba con los rendimientos en moneda local muestran evidencia de contagio únicamente para los rendimientos del mercado accionario de Argentina (MERVAL) mientras que los rendimientos del mercado accionario de Brasil (IBOV) aparentemente se contagiaron a partir del resultado de la prueba asintótica, no obstante, tal resultado se descarta a partir del valor- $p$ resultante del método con bootstrap. Mientras que cuando se toma como variable dependiente a los rendimientos accionarios del DJIA y del S\&P 500, los resultados son prácticamentelos mismos ya que únicamente hay evidencia de contagio para el MERVAL al nivel de significancia del $5 \%$ y del $10 \%$ para el IBOVESPA con respecto al DJIA.

En tanto que los resultados de la prueba efectuada con los rendimientos accionarios medidos en dólares, presentados en las tablas 4, 5 y 6 tomando como variable independiente a los rendimientos accionarios del NASDAQ, DJIA y del S\&P 500, respectivamente, muestran evidencia de contagio para los mercados de Argentina (MERVAL) y de Brasil (IBOV) al 5\% y para México (IPyC) al 10\%, con los rendimientos del NASDAQ. No obstante, cuando se toma como variable independientea los rendimientos del DJIA este sólo muestra evidencia de contagio para el MERVAL al nivel de significancia del 5\% y del 10\% para el IBOV, en tanto que con respecto a los rendimientos del S\&P 500 únicamente hay evidencia de contagio para el MERVAL como se puede apreciar en la tabla 6. 
Tabla 1

Resultados de las pruebas de contagio para los países de América Latina.

Datos con moneda local (Variable independiente: Rendimientos del NASDAQ)

Estimaciones con Bootstrap

Valor-p

\begin{tabular}{|c|c|c|c|c|c|}
\hline Variable & OLS & Media & Mediana & Asintótico & Bootstrap \\
\hline \multicolumn{6}{|c|}{ IPSA } \\
\hline Intercepto $\left(\alpha_{1}\right)$ & -0.0418 & -0.0415 & -0.0418 & 0.6288 & 0.5933 \\
\hline Cambio en el intercepto $\left(\alpha_{2}\right)$ & 0.1461 & -0.1439 & -0.1423 & 0.2573 & 0.2792 \\
\hline Pendiente $\left(\beta_{1}\right)$ & 0.4010 & 0.0000 & 0.4017 & 0.0000 & 0.0000 \\
\hline Cambio en la pendiente $\left(\beta_{2}\right)$ & -0.0385 & -0.0407 & -0.0424 & 0.5623 & 0.6073 \\
\hline \multicolumn{6}{|c|}{ IGBC } \\
\hline Intercepto $\left(\alpha_{1}\right)$ & -0.0233 & -0.0236 & -0.0231 & 0.8160 & 0.7986 \\
\hline Cambio en el intercepto $\left(\alpha_{2}\right)$ & 0.0388 & 0.0386 & 0.0381 & 0.7951 & 0.7983 \\
\hline Pendiente $\left(\beta_{1}\right)$ & 0.1497 & 0.0000 & 0.1487 & 0.0286 & 0.0067 \\
\hline Cambio en la pendiente $\left(\beta_{2}\right)$ & 0.0624 & 0.0635 & 0.0630 & 0.4175 & 0.3808 \\
\hline \multicolumn{6}{|c|}{ MERVAL } \\
\hline Intercepto $\left(\alpha_{1}\right)$ & -0.0990 & -0.0989 & -0.0989 & 0.4648 & 0.2527 \\
\hline Cambio en el intercepto $\left(\alpha_{2}\right)$ & 0.1622 & 0.1645 & 0.1662 & 0.4216 & 0.4419 \\
\hline Pendiente $\left(\beta_{1}\right)$ & 0.3519 & 0.0000 & 0.3521 & 0.0002 & 0.0000 \\
\hline Cambio en la pendiente $\left(\beta_{2}\right)$ & 0.3538 & 0.3560 & 0.3569 & 0.0007 & 0.0013 \\
\hline \multicolumn{6}{|c|}{ IBOV } \\
\hline Intercepto $\left(\alpha_{1}\right)$ & -0.0114 & -0.0113 & -0.0111 & 0.9279 & 0.9180 \\
\hline Cambio en el intercepto $\left(\alpha_{2}\right)$ & 0.1078 & 0.1036 & 0.1040 & 0.5652 & 0.5915 \\
\hline Pendiente $\left(\beta_{1}\right)$ & 0.7278 & 0.0000 & 0.7299 & 0.0000 & 0.0000 \\
\hline Cambio en la pendiente $\left(\beta_{2}\right)$ & 0.1966 & 0.1948 & 0.1946 & 0.0421 & 0.0500 \\
\hline \multicolumn{6}{|c|}{ IPyC } \\
\hline Intercepto $\left(\alpha_{1}\right)$ & -0.0369 & -0.0373 & -0.0383 & 0.6760 & 0.5797 \\
\hline Cambio en el intercepto $\left(\alpha_{2}\right)$ & 0.0913 & -0.0889 & 0.0870 & 0.4877 & 0.5259 \\
\hline Pendiente $\left(\beta_{1}\right)$ & 0.6581 & 0.0000 & 0.6594 & 0.0000 & 0.0000 \\
\hline Cambio en la pendiente $\left(\beta_{2}\right)$ & 0.0453 & 0.0442 & 0.0451 & 0.5037 & 0.5285 \\
\hline
\end{tabular}

Nota: Las estimaciones se obtuvieron a través del código GAUSS desarrollado por Hacker y Hatemi-J (2009) el cual está disponible en línea. 
Tabla 2

Resultados de las pruebas de contagio para los países de América Latina.

Datos con moneda local (Variable independiente: Rendimientos del DJIA)

Estimaciones con Bootstrap

Valor-p

\begin{tabular}{|c|c|c|c|c|c|}
\hline \multirow{2}{*}{ Variable } & \multirow[b]{2}{*}{ OLS } & & \\
\hline & & Media & Mediana & Asintótico & Bootstrap \\
\hline \multicolumn{6}{|c|}{ IPSA } \\
\hline Intercepto $\left(\alpha_{1}\right)$ & -0.0356 & -0.0352 & -0.0350 & 0.6742 & 0.6512 \\
\hline Cambio en el intercepto $\left(\alpha_{2}\right)$ & 0.1609 & 0.1591 & 0.1567 & 0.2031 & 0.2286 \\
\hline Pendiente $\left(\beta_{1}\right)$ & 0.4723 & 0.0000 & 0.4730 & 0.0000 & 0.0000 \\
\hline Cambio en la pendiente $\left(\beta_{2}\right)$ & -0.0494 & -0.0515 & -0.0536 & 0.5091 & 0.5568 \\
\hline \multicolumn{6}{|c|}{ IGBC } \\
\hline Intercepto $\left(\alpha_{1}\right)$ & -0.0194 & -0.0198 & -0.0195 & 0.8444 & 0.8274 \\
\hline Cambio en el intercepto $\left(\alpha_{2}\right)$ & 0.0504 & 0.0518 & 0.0513 & 0.7320 & 0.7259 \\
\hline Pendiente $\left(\beta_{1}\right)$ & 0.1953 & 0.0000 & 0.1941 & 0.0129 & 0.0008 \\
\hline Cambio en la pendiente $\left(\beta_{2}\right)$ & 0.0741 & 0.0757 & 0.0748 & 0.3958 & 0.3399 \\
\hline \multicolumn{6}{|c|}{ MERVAL } \\
\hline Intercepto $\left(\alpha_{1}\right)$ & -0.0960 & -0.0954 & -0.0950 & 0.4795 & 0.2831 \\
\hline Cambio en el intercepto $\left(\alpha_{2}\right)$ & 0.1931 & 0.1974 & 0.2002 & 0.3400 & 0.3644 \\
\hline Pendiente $\left(\beta_{1}\right)$ & 0.3855 & 0.0000 & 0.3856 & 0.0004 & 0.0000 \\
\hline Cambio en la pendiente $\left(\beta_{2}\right)$ & 0.3918 & 0.3963 & 0.3956 & 0.0012 & 0.0023 \\
\hline \multicolumn{6}{|c|}{ IBOV } \\
\hline Intercepto $\left(\alpha_{1}\right)$ & -0.0056 & -0.0051 & -0.0041 & 0.9654 & 0.9735 \\
\hline Cambio en el intercepto $\left(\alpha_{2}\right)$ & 0.1443 & 0.1403 & 0.1413 & 0.4526 & 0.4811 \\
\hline Pendiente $\left(\beta_{1}\right)$ & 0.7929 & 0.0000 & 0.7939 & 0.0000 & 0.0000 \\
\hline Cambio en la pendiente $\left(\beta_{2}\right)$ & 0.2110 & 0.2091 & 0.2108 & 0.0643 & 0.0944 \\
\hline \multicolumn{6}{|c|}{ IPyC } \\
\hline Intercepto $\left(\alpha_{1}\right)$ & -0.0268 & -0.0273 & -0.0275 & 0.7646 & 0.6966 \\
\hline Cambio en el intercepto $\left(\alpha_{2}\right)$ & 0.1134 & 0.1110 & 0.1102 & 0.3952 & 0.4301 \\
\hline Pendiente $\left(\beta_{1}\right)$ & 0.7744 & 0.0000 & 0.7739 & 0.0000 & 0.0000 \\
\hline Cambio en la pendiente $\left(\beta_{2}\right)$ & -0.0101 & -0.0109 & -0.0104 & 0.8981 & 0.8956 \\
\hline
\end{tabular}

Nota: Las estimaciones se obtuvieron a través del código GAUSS desarrollado por Hacker y Hatemi-J (2009) el cual está disponible en línea. 
Tabla 3

Resultados de las pruebas de contagio para los países de América Latina.

Datos con moneda local (Variable independiente: Rendimientos del S\&P 500)

\begin{tabular}{|c|c|c|c|c|c|}
\hline \multirow[b]{2}{*}{ Variable } & \multirow[b]{2}{*}{ OLS } & \multicolumn{2}{|c|}{ Estimaciones con Bootstrap } & \multicolumn{2}{|c|}{ Valor-p } \\
\hline & & Media & Mediana & Asintótico & Bootstrap \\
\hline \multicolumn{6}{|c|}{ IPSA } \\
\hline Intercepto $\left(\alpha_{1}\right)$ & -0.0343 & -0.0336 & -0.0336 & 0.6845 & 0.6630 \\
\hline Cambio en el intercepto $\left(\alpha_{2}\right)$ & 0.1576 & 0.1552 & 0.1526 & 0.2108 & 0.2353 \\
\hline Pendiente $\left(\beta_{1}\right)$ & 0.4607 & 0.0000 & 0.4618 & 0.0000 & 0.0000 \\
\hline Cambio en la pendiente $\left(\beta_{2}\right)$ & -0.0788 & -0.0809 & -0.0823 & 0.2627 & 0.3117 \\
\hline \multicolumn{6}{|c|}{ IGBC } \\
\hline Intercepto $\left(\alpha_{1}\right)$ & -0.0180 & -0.0183 & -0.0181 & 0.8546 & 0.8401 \\
\hline Cambio en el intercepto $\left(\alpha_{2}\right)$ & 0.0486 & 0.0490 & 0.0491 & 0.7401 & 0.7333 \\
\hline Pendiente $\left(\beta_{1}\right)$ & 0.1995 & 0.0000 & 0.1980 & 0.0074 & 0.0006 \\
\hline Cambio en la pendiente $\left(\beta_{2}\right)$ & 0.0489 & 0.0499 & 0.0497 & 0.5503 & 0.4893 \\
\hline \multicolumn{6}{|c|}{ MERVAL } \\
\hline Intercepto $\left(\alpha_{1}\right)$ & -0.0910 & -0.0903 & -0.0900 & 0.4888 & 0.2973 \\
\hline Cambio en el intercepto $\left(\alpha_{2}\right)$ & 0.1903 & 0.1929 & 0.1947 & 0.3316 & 0.3587 \\
\hline Pendiente $\left(\beta_{1}\right)$ & 0.4195 & 0.0000 & 0.4189 & 0.0000 & 0.0000 \\
\hline Cambio en la pendiente $\left(\beta_{2}\right)$ & 0.3181 & 0.3211 & 0.3207 & 0.0038 & 0.0041 \\
\hline \multicolumn{6}{|c|}{ IBOV } \\
\hline Intercepto $\left(\alpha_{1}\right)$ & 0.0019 & 0.0022 & 0.0023 & 0.9880 & 0.9842 \\
\hline Cambio en el intercepto $\left(\alpha_{2}\right)$ & 0.1355 & 0.1306 & 0.1321 & 0.4636 & 0.4961 \\
\hline Pendiente $\left(\beta_{1}\right)$ & 0.8319 & 0.0000 & 0.8325 & 0.0000 & 0.0000 \\
\hline Cambio en la pendiente $\left(\beta_{2}\right)$ & 0.0951 & 0.0933 & 0.0948 & 0.3568 & 0.3693 \\
\hline \multicolumn{6}{|c|}{ IPyC } \\
\hline Intercepto $\left(\alpha_{1}\right)$ & -0.0241 & -0.0243 & -0.0245 & 0.7801 & 0.7205 \\
\hline Cambio en el intercepto $\left(\alpha_{2}\right)$ & 0.1096 & 0.1062 & 0.1055 & 0.3942 & 0.4300 \\
\hline Pendiente $\left(\beta_{1}\right)$ & 0.7614 & 0.0000 & 0.7614 & 0.0000 & 0.0000 \\
\hline Cambio en la pendiente $\left(\beta_{2}\right)$ & -0.0562 & -0.0571 & -0.0556 & 0.4340 & 0.4214 \\
\hline
\end{tabular}

Nota: Las estimaciones se obtuvieron a través del código GAUSS desarrollado por Hacker y Hatemi-J (2009) el cual está disponible en línea. 
Tabla 4

Resultados de las pruebas de contagio para los países de América Latina.

Datos en dólares (Variable independiente: Rendimientos del NASDAQ)

\begin{tabular}{|c|c|c|c|c|c|}
\hline \multicolumn{4}{|c|}{ Estimaciones con Bootstrap } & \multicolumn{2}{|c|}{ Valor-p } \\
\hline Variable & OLS & Media & Mediana & Asintótico & Bootstrap \\
\hline \multicolumn{6}{|c|}{ IPSA } \\
\hline Intercepto $\left(\alpha_{1}\right)$ & -0.0752 & -0.0742 & -0.0744 & 0.5003 & 0.4138 \\
\hline Cambio en el intercepto $\left(\alpha_{2}\right)$ & 0.1472 & 0.1462 & 0.1465 & 0.3763 & 0.3823 \\
\hline Pendiente $\left(\beta_{1}\right)$ & 0.3589 & 0.0000 & 0.3582 & 0.0000 & 0.0000 \\
\hline Cambio en la pendiente $\left(\beta_{2}\right)$ & -0.0752 & -0.0742 & -0.0744 & 0.5003 & 0.4138 \\
\hline \multicolumn{6}{|c|}{ IGBC } \\
\hline Intercepto $\left(\alpha_{1}\right)$ & -0.0442 & -0.0438 & -0.0427 & 0.7155 & 0.6925 \\
\hline Cambio en el intercepto $\left(\alpha_{2}\right)$ & 0.0707 & 0.0709 & 0.0706 & 0.6956 & 0.6950 \\
\hline Pendiente $\left(\beta_{1}\right)$ & 0.1684 & 0.0000 & 0.1685 & 0.0416 & 0.0088 \\
\hline Cambio en la pendiente $\left(\beta_{2}\right)$ & -0.0040 & -0.0020 & -0.0012 & 0.9657 & 0.9884 \\
\hline \multicolumn{6}{|c|}{ MERVAL } \\
\hline Intercepto $\left(\alpha_{1}\right)$ & -0.0877 & -0.0876 & -0.0878 & 0.5343 & 0.3290 \\
\hline Cambio en el intercepto $\left(\alpha_{2}\right)$ & 0.0491 & 0.0518 & -0.0542 & 0.8155 & 0.8085 \\
\hline Pendiente $\left(\beta_{1}\right)$ & 0.3606 & 0.0000 & 0.3612 & 0.0002 & 0.0000 \\
\hline Cambio en la pendiente $\left(\beta_{2}\right)$ & -0.0877 & -0.0876 & -0.0878 & 0.5343 & 0.3290 \\
\hline \multicolumn{6}{|c|}{ IBOV } \\
\hline Intercepto $\left(\alpha_{1}\right)$ & -0.0047 & -0.0041 & -0.0018 & 0.9794 & 0.9901 \\
\hline Cambio en el intercepto $\left(\alpha_{2}\right)$ & 0.0815 & 0.0763 & 0.0749 & 0.7647 & 0.7897 \\
\hline Pendiente $\left(\beta_{1}\right)$ & 0.8043 & 0.0000 & 0.8060 & 0.0000 & 0.0000 \\
\hline Cambio en la pendiente $\left(\beta_{2}\right)$ & 0.3121 & 0.3087 & 0.3113 & 0.0265 & 0.0368 \\
\hline \multicolumn{6}{|c|}{ IPyC } \\
\hline Intercepto $\left(\alpha_{1}\right)$ & -0.0239 & -0.0245 & -0.0254 & 0.8493 & 0.7344 \\
\hline Cambio en el intercepto $\left(\alpha_{2}\right)$ & -0.0199 & -0.0222 & -0.0236 & 0.9153 & 0.9085 \\
\hline Pendiente $\left(\beta_{1}\right)$ & 0.7082 & 0.0000 & 0.7098 & 0.0000 & 0.0000 \\
\hline Cambio en la pendiente $\left(\beta_{2}\right)$ & 0.1648 & 0.1631 & 0.1627 & 0.0877 & 0.0920 \\
\hline
\end{tabular}

Nota: Las estimaciones se obtuvieron a través del código GAUSS desarrollado por Hacker y Hatemi-J (2009) el cual está disponible en línea. 
Tabla 5

Resultados de las pruebas de contagio para los países de América Latina.

Datos en dólares (Variable independiente: Rendimientos del DJIA)

\begin{tabular}{|c|c|c|c|c|c|}
\hline & & \multicolumn{2}{|c|}{ Estimaciones con Bootstrap } & \multicolumn{2}{|c|}{ Valor-p } \\
\hline Variable & OLS & Media & Mediana & Asintótico & Bootstrap \\
\hline \multicolumn{6}{|c|}{ IPSA } \\
\hline Intercepto $\left(\alpha_{1}\right)$ & -0.0680 & -0.0669 & -0.0655 & 0.5374 & 0.4673 \\
\hline Cambio en el intercepto $\left(\alpha_{2}\right)$ & 0.1592 & 0.1596 & 0.1603 & 0.3326 & 0.3382 \\
\hline Pendiente $\left(\beta_{1}\right)$ & 0.4427 & 0.0000 & 0.4426 & 0.0000 & 0.0000 \\
\hline Cambio en la pendiente $\left(\beta_{2}\right)$ & -0.0529 & -0.0529 & -0.0537 & 0.5870 & 0.6190 \\
\hline \multicolumn{6}{|c|}{ IGBC } \\
\hline Intercepto $\left(\alpha_{1}\right)$ & -0.0383 & -0.0380 & -0.0368 & 0.7500 & 0.7289 \\
\hline Cambio en el intercepto $\left(\alpha_{2}\right)$ & 0.0778 & 0.0797 & 0.0795 & 0.6638 & 0.6590 \\
\hline Pendiente $\left(\beta_{1}\right)$ & 0.2370 & 0.0000 & 0.2361 & 0.0131 & 0.0015 \\
\hline Cambio en la pendiente $\left(\beta_{2}\right)$ & -0.0215 & -0.0203 & -0.0196 & 0.8397 & 0.8382 \\
\hline \multicolumn{6}{|c|}{ MERVAL } \\
\hline Intercepto $\left(\alpha_{1}\right)$ & -0.0848 & -0.0841 & -0.0845 & 0.5495 & 0.3533 \\
\hline Cambio en el intercepto $\left(\alpha_{2}\right)$ & 0.0792 & 0.0843 & 0.0862 & 0.7076 & 0.7092 \\
\hline Pendiente $\left(\beta_{1}\right)$ & 0.3930 & 0.0000 & 0.3933 & 0.0005 & 0.0000 \\
\hline Cambio en la pendiente $\left(\beta_{2}\right)$ & 0.3709 & 0.3761 & 0.3747 & 0.0032 & 0.0062 \\
\hline \multicolumn{6}{|c|}{ IBOV } \\
\hline Intercepto $\left(\alpha_{1}\right)$ & 0.0006 & 0.0020 & 0.0037 & 0.9975 & 0.9979 \\
\hline Cambio en el intercepto $\left(\alpha_{2}\right)$ & 0.1293 & 0.1234 & 0.1224 & 0.6382 & 0.6785 \\
\hline Pendiente $\left(\beta_{1}\right)$ & 0.8634 & 0.0000 & 0.8645 & 0.0000 & 0.0000 \\
\hline Cambio en la pendiente $\left(\beta_{2}\right)$ & 0.3631 & 0.3607 & 0.3632 & 0.0263 & 0.0532 \\
\hline \multicolumn{6}{|c|}{ IPyC } \\
\hline Intercepto $\left(\alpha_{1}\right)$ & -0.0132 & -0.0139 & -0.0136 & 0.9174 & 0.8554 \\
\hline Cambio en el intercepto $\left(\alpha_{2}\right)$ & 0.0086 & 0.0067 & 0.0053 & 0.9640 & 0.9802 \\
\hline Pendiente $\left(\beta_{1}\right)$ & 0.8302 & 0.0000 & 0.8301 & 0.0000 & 0.0000 \\
\hline Cambio en la pendiente $\left(\beta_{2}\right)$ & 0.1127 & 0.1119 & 0.1125 & 0.3180 & 0.3162 \\
\hline
\end{tabular}

Nota: Las estimaciones se obtuvieron a través del código GAUSS desarrollado por Hacker y Hatemi-J (2009) el cual está disponible en línea. 
Tabla 6

Resultados de las pruebas de contagio para los países de América Latina.

Datos en dólares (Variable independiente: Rendimientos del S\&P 500)

\begin{tabular}{|c|c|c|c|c|c|}
\hline \multirow[b]{2}{*}{ Variable } & \multirow[b]{2}{*}{ OLS } & \multicolumn{2}{|c|}{ Estimaciones con Bootstrap } & \multicolumn{2}{|c|}{ Valor- $p$} \\
\hline & & Media & Mediana & Asintótico & Bootstrap \\
\hline \multicolumn{6}{|c|}{ IPSA } \\
\hline Intercepto $\left(\alpha_{1}\right)$ & -0.0683 & -0.0668 & -0.0669 & 0.5336 & 0.4641 \\
\hline Cambio en el intercepto $\left(\alpha_{2}\right)$ & 0.1595 & 0.1587 & 0.1593 & 0.3293 & 0.3389 \\
\hline Pendiente $\left(\beta_{1}\right)$ & 0.4152 & 0.0000 & 0.4147 & 0.0000 & 0.0000 \\
\hline Cambio en la pendiente $\left(\beta_{2}\right)$ & -0.0522 & -0.0524 & -0.0528 & 0.5674 & 0.5860 \\
\hline \multicolumn{6}{|c|}{ IGBC } \\
\hline Intercepto $\left(\alpha_{1}\right)$ & -0.0394 & -0.0391 & -0.0381 & 0.7425 & 0.7220 \\
\hline Cambio en el intercepto $\left(\alpha_{2}\right)$ & 0.0791 & 0.0800 & 0.0792 & 0.6582 & 0.6569 \\
\hline Pendiente $\left(\beta_{1}\right)$ & 0.2111 & 0.0000 & 0.2107 & 0.0201 & 0.0026 \\
\hline Cambio en la pendiente $\left(\beta_{2}\right)$ & -0.0094 & -0.0083 & -0.0078 & 0.9250 & 0.9277 \\
\hline \multicolumn{6}{|c|}{ MERVAL } \\
\hline Intercepto $\left(\alpha_{1}\right)$ & -0.0795 & -0.0795 & -0.0787 & 0.5625 & 0.3765 \\
\hline Cambio en el intercepto $\left(\alpha_{2}\right)$ & 0.0766 & 0.0766 & 0.0798 & 0.7081 & 0.7098 \\
\hline Pendiente $\left(\beta_{1}\right)$ & 0.4294 & 0.4295 & 0.0000 & 0.0000 & 0.0000 \\
\hline Cambio en la pendiente $\left(\beta_{2}\right)$ & 0.2987 & 0.2987 & 0.3020 & 0.0092 & 0.0091 \\
\hline \multicolumn{6}{|c|}{ IBOV } \\
\hline Intercepto $\left(\alpha_{1}\right)$ & 0.0098 & 0.0109 & 0.0133 & 0.9563 & 0.9195 \\
\hline Cambio en el intercepto $\left(\alpha_{2}\right)$ & 0.1183 & 0.1116 & 0.1094 & 0.6586 & 0.7042 \\
\hline Pendiente $\left(\beta_{1}\right)$ & 0.9185 & 0.0000 & 0.9194 & 0.0000 & 0.0000 \\
\hline Cambio en la pendiente $\left(\beta_{2}\right)$ & 0.2133 & 0.2109 & 0.2134 & 0.1543 & 0.1857 \\
\hline \multicolumn{6}{|c|}{ IPyC } \\
\hline Intercepto $\left(\alpha_{1}\right)$ & -0.0104 & -0.0108 & -0.0112 & 0.9335 & 0.8791 \\
\hline Cambio en el intercepto $\left(\alpha_{2}\right)$ & 0.0049 & 0.0015 & 0.0002 & 0.9789 & 0.9994 \\
\hline Pendiente $\left(\beta_{1}\right)$ & 0.8162 & 0.0000 & 0.8159 & 0.0000 & 0.0000 \\
\hline Cambio en la pendiente $\left(\beta_{2}\right)$ & 0.0570 & 0.0558 & 0.0570 & 0.5813 & 0.5544 \\
\hline
\end{tabular}

Nota: Las estimaciones se obtuvieron a través del código GAUSS desarrollado por Hacker y Hatemi-J (2009) el cual está disponible en línea. 
De los resultados de las pruebas de contagio efectuadas de los mercados accionarios más representativos de Estados Unidos hacia los principales mercados accionarios de América Latina destaca el hecho de que casi la totalidad de los interceptos estimados $\left(\alpha_{1}\right)$ resultaron negativos, con excepción del mercado de Brasil (IBOV) cuando se relacionan con el S\&P 500 con los datos en moneda local y con el DJIA y el S\&P 500 en dólares, sugiriendo de este modo que la mayoría de los rendimientos promedio estimados bajo el periodo de estudio son negativos, no obstante ninguno resultó significativo.Algo similar ocurre con el coeficiente que mide el cambio en el intercepto $\left(\alpha_{2}\right)$ que en su mayoría es positivo en ningún caso resultó estadísticamente significativo. Sin embargo, un hecho interesante es que el coeficiente que mide la pendiente $\left(\beta_{1}\right)$, independientemente de que rendimientos accionarios sea considerados como variable independiente, resultó positivo y significativo, lo cual se puede interpretar como evidencia de unainterdependencia generalizada de los rendimientos de los mercados accionarios de los mercados considerados en el análisis. En tanto que los coeficientes estimados del cambio en la pendiente $\left(\beta_{2}\right)$ resultaron positivos y negativos y cuya significancia fue evaluada con el fin de probar la hipótesis de contagio como se discutió anteriormente.

Otro resultado que es preciso enfatizar es el hecho de que los resultados de la prueba de contagio son prácticamente los mismos en comparación a la inferencia que se puede extraer de las pruebas $t$ asintóticas, con excepción de la pruebas efectuadas para el IBOVESPA cuando se relaciona con el NASDAQ, con los rendimientos en moneda local, y con el DJIA, con los rendimientos medidos en dólares, ya que en ambos casos el resultado de la prueba de contagio cambia de aparentemente tener evidencia de contagio con la prueba asintótica a rechazarla con el método de bootstrap.

Por último, es importante tomar en cuenta que el tipo de cambio no parece desempeñar un papel importante en la especificación de las pruebas de contagio financiero entre los rendimientos de los mercados accionarios en virtud de que los resultados no se modifican ya sea que se considere a dichos rendimientos en moneda local o en dólares.

\section{Conclusiones}

En este trabajo empleamos la prueba de contagio financiero propuesta por Hatemi y Hacker (2005) para probar contagio en los principales mercados accionarios de América Latina por parte de los mercados accionarios de los Estados Unidos, la cual consiste en determinar sí el coeficiente de la pendiente cambia en una regresión de los rendimientos accionarios de un país en los rendimientos accionarios de otro país. En tal prueba hay evidencia de contagio si el coeficiente de la pendiente aumenta durante el periodo de crisis.

Hatemi y Hacker (2005) concluyeron que en dicha prueba no es posible obtener inferencia valida si está se basa sobre la distribución asintótica ${ }^{t}$ por lo que sugirieron un enfoque 
alternativo que se basa en un remuestreo a través de bootstraping, el cual sostienen posee mejores propiedades, siendo una de sus principales cualidades la de ser robusto en presencia de heterocedasticidad y falta de normalidad en los residuos del modelo estimado, y este método es el que hemos empleado en este trabajo.

El coeficiente beta estimado de la pendiente resultó estadísticamente significativo en todos los modelos en los que se llevó a cabo la prueba, lo cual sugiere que hay una permanente dependencia de los mercados latinoamericanos que fueron considerados con respecto a los principales índices accionarios de los Estados Unidos.

Sin embargo, se encontró evidencia de contagio financiero de la crisis subprimecon los rendimientos medidos en dólares en el mercado argentino (MERVAL) al nivel de significancia del $5 \%$ con respecto a los tres mercados accionarios de Estados Unidos considerados. En el brasileño (IBOVESPA) al nivel de significancia del 10\% con respecto al NASDAQ y al DJIA.

Resultados similares se encontraron con los rendimientos medidos en dólares ya que también se encontró evidencia de contagio para el MERVAL y el IBOVESPA proveniente del NASDAQ y del DJIA al nivel de significancia del 5\%, para México (IPyC) proveniente del NASDAQ al nivel de significancia del 10\% y para Argentina del S\&P 500 también al nivel de significancia del $5 \%$.

De esta forma, los resultados de la prueba de contagio empleada revelan evidencia de contagio en los mercados de Argentina y de Brasil, en el caso del primero la evidencia es mayor ya que independientemente del índice accionario de Estados Unidos que se considere en la prueba el resultado es el mismo, mientras que para Brasil el resultado depende en buena medida del índice accionario de Estados Unidos.Paradójicamente en el caso de México, y a pesar de su alto grado de integración comercial con su vecino país del norte,la evidencia de contagio es escasa con esta prueba y se encontró con respecto al índice menos representativo de los tres considerados para Estados Unidos.

Estos resultados son consistentes con lo reportado en otros estudios y con otras metodologías para Argentina y Brasil, de acuerdo con Naoui, Liouane y Brahim (2010) pero no para México. Los resultados son consistentes también en el caso de Argentina y Brasil de acuerdo a lo encontrado por Bejarano, Gómez, Melo y Torres (2015) pero no para Brasil, Chile y México.

Los anteriores resultados sugieren evidencia de contagio en los mercados accionarios de Argentina y Brasil por parte del mercado accionario de Estados Unidos. Lo cual puede deberse a la forma en la cual se encuentran integrados o interrelacionados los mercados y a la manera en la cual rápidamente es procesada la información en los mercados financieros. No obstante, queda pendiente abordar algunas otras posibles interrelaciones entre estos mercados que revelen la forma en que se propaga el contagio financiero entre los mercados accionarios de los principales mercados accionarios a los mercados emergentes. 


\section{Referencias}

Agosin, M. y French-Davis, R. (1993). La liberalización comercial en América Latina. Revista de la CEPAL, 50, 41-62.

Bekaert,G., Harvey, C.R., y Lumsdaine, R. L. (2002). Dating the integration of world equity markets. Journal of Financial Economics, 65, 203-247. https://doi.org/10.1016/S0304-405X(02)00139-3

Bekaert G., Harvey C. y Lundblad C. (2005). Does financial liberalisation spur growth? Journal of Financial Economics, 77(1), pp. 3-55. doi:10.1016/j.jfineco.2004.05.007

Bejarano-Bejarano, L. V., Gomez-Gonzalez, J. E., Melo-Velandia, L. F., y Torres-Gorron, J. E. (2015). Financial Contagion in Latin America (No. 884). Borradores de Economía. Banco de la República.

Bodart, V. y Candelon, B. (2009). Evidence of interdependence and contagion using a frequency domain framework. Emerging Markets Review, 10, 140-150. https://doi.org/10.1016/j.ememar.2008.11.003

Breitung, J. y Candelon, B. (2006). Testing for short and long-run causality: a frequency domain approach. Journal of Econometrics, 12, 363-378. https://doi.org/10.1016/j.jeconom.2005.02.004

Calvo, S. y Reinhart, C. M. (1996). Capital flows to Latin America: is there evidence of contagion effects?, in Private Capital Flows to Emerging Markets After the Mexican Crisis. (Eds) G. A. Calvo, M. Goldstein and E. Hochreiter, Institute for International Economics, Washington, DC.

Collins D. y Biekpe N. (2003). Contagion: a fear for African equity markets?. Journal of Economics \& Business, 55(5), pp. 405-426. doi:10.1016/S0148-6195(03)00020-1

Chirinos G., M. (2013). Medición de contagio e interdependencia financieros mediante cópulas y eventos extremos en los países de la América Latina. El Trimestre Económico. Vol. LXXX (1), núm. 317, pp. 169-206. http:// dx.doi.org/10.20430/ete.v80i317.86

Dimitriou, D., Kenourgios, D. y Simos, T. (2013). Global financial crisis and emerging stock market contagion: A multivariate FIAPARCH-DCC approach. International Review of Financial Analysis, 30, 46-56. https://doi. org/10.1016/j.irfa.2013.05.008

Dooley, M. y Hutchison, M. (2009). Transmission of the U.S. subprime crisis to emerging markets: Evidence on the decoupling-recoupling hypothesis. Journal of International Money and Finance, 28, 1331-1349. https:// doi.org/10.1016/j.jimonfin.2009.08.004

Erdal, F. y L. Gunduz (2001). An Empirical Investigation of the Interdependence of Istanbul Stock Exchange with Selected Stock Markets. An International Conference: Economies and Business in Transition, Global Business and Technology Association, Istanbul.

Fedderke, J. W. y Marinkov, M. (2016). Diagnosing the Source of Financial Market Shocks: An Application to the Asian, Subprime and European Financial Crises. Pacific Economic Review, doi: 10.1111/1468-0106.12162.

Forbes, K. y Rigobon, R. (2000). Contagion in Latin America: definitions, measurement, and policy implications. NBER Working Paper, p. 7885.

Forbes, K. J. y Rigobon, R. (2002). No contagion, only interdependence: measuring stock market comovements. Journal of Finance, LVII, pp. 2223-61. https://doi.org/10.1111/0022-1082.00494

Fuchun, L. (2009). Testing for financial contagion with applications to the Canadian banking system. Bank of Canada, Working papers 09-14

Glick, R. y Rose, A.K. (1999). Contagion and crisis. Why are currency crises regional? Journal of International Money and Finance, 18, 603-617.

Hacker, R.S. y Hatemi-J, A. (2009). ContagT: GAUSS module to implement a pairwise bootstrap test for contagion. Statistical Software Components, G00007. Boston College Department of Economics (Available online).

Hatemi-J, A. y Hacker, R.S. (2005). An alternative method to test for contagion with an application to the Asian financial crisis. Appl. Financ. Econ. Lett. 1 (6), 343-347. https://doi.org/10.1080/17446540500393468 
Hatemi-J, A., Roca, E. y Al-Shayeb, A. (2014). How Integrated are Real Estate Markets with the World Market? Evidence from Case-Wise Bootstrap Analysis. Economic Modelling, Volume 37, pp. 137-142. https://doi. org/10.1016/j.econmod.2013.10.037

Hinich, J. M. (1996). Testing for Dependence in the input to a linear time series model. Nonparametric Statistics, 6, 205-221. https://doi.org/10.1080/10485259608832672

Hon, M. T., Strauss, J. y Yong, S. K. (2004). Contagion in financial markets after September 11: myth or reality?. Journal of Financial Research, 27, pp. 95-114. https://doi.org/10.1111/j.1475-6803.2004.00079.x

Horta P., Mendes C. y Vieira I. (2008). Contagion effects of the US subprime crisis on developed countries. CEFAGE-UE Working Paper 2008/08.

Kaminsky, G., Reinhart, C. (2000). On crises, contagion, and confusion. Journal of International Economics, 51, 145-168. https://doi.org/10.1016/S0022-1996(99)00040-9

Khallouli, Wajih y René Sandretto (2012).Testing for "Contagion" of the Subprime Crisis on the Middle East and North African Stock Markets: A Markov Switching EGARCH Approach. Journal of Economic Integration. Vol. 27, No. 1, pp. 134-166.

King, M. A. y Wadhwani, S. (1990). Transmission of volatility between stock markets. Review of Financial Studies, 3, 5-33.

Lee, S. B. y Kim, J. K. (1993). Does the October 1987 crash strengthen the co-movements among national stock markets?. Review of Financial Economics, 3, 89-102. https://doi.org/10.1002/j.1873-5924.1993.tb00574.x

Lee, H.Y. (2012). Contagion in international stock markets during the subprime mortgage crisis. International Journal of Economics and Financial Issues, 2: 41-53

Longstaff, F.A. (2010). The subprime credit crisis and contagion in financial markets. Journal of Financial Economics, 97 (3), 436-450. https://doi.org/10.1016/j.jfineco.2010.01.002

Mollah, S., Quoreshi, A.M.M.S. y Zafirov, G.(2016). Equity market contagion during global financial and eurozone crises: evidence from a dynamic correlationanalysis. J. Int. Financ. Markets Inst. Money 41, 151-167.

Naoui, K., Liouane, N. y Brahim, S. (2010). A dynamic conditional correlation analysis of financial contagion: the case of the subprime credit crisis. International Journal of Economics and Finance 2 (3), 85-96.

Neaime, S. (2002). Liberalization and Financial Integration of MENA Stock Markets. Paper presented at the 9th Economic Research Forum Meeting, American University in Sharjah, United Arab Emirates.

Polanco J., Fernández-Macho J., Neumann M. y Faria S. (2018). A pre-crisis vs. crisis analysis of peripheral EU stock markets by means of wavelet transform and a nonlinear causality test. Physica A, pp. 1211-1227. https:// doi.org/10.1016/j.physa.2017.08.065

Pretorius, A. y Beer, J. (2004). Contagion in Africa: South Africa and a troubled neighbour, Zimbabwe. Economic Modelling, 27, 95-114. https://doi.org/10.1016/j.econmod.2003.09.007

Romero-Meza, R., Bonilla, C., Benedetti, H., y Serletis, A. (2015). Nonlinearities and Financial Contagion in Latin American Stock Markets. Economic Modelling.51, 653-656. https://doi.org/10.1016/j.econmod.2015.09.012

Wang, G.-J., Xie, C., Lin, M., y Stanley, H. E. (2017). Stock market contagion during the global financial crisis: A multiscale approach. Finance Research Letters, 22, pp. 163-168. https://doi.org/10.1016/j.frl.2016.12.025 